\title{
Tetramer Bound States in Heteronuclear Systems
}

\author{
C. H. Schmickler用 \\ Institut für Kernphysik, Technische Universität Darmstadt, 64289 Darmstadt, Germany and \\ RIKEN Nishina Center, RIKEN, Saitama 351-0198, Japan \\ H.-W. Hammer \\ Institut für Kernphysik, Technische Universität Darmstadt, 64289 Darmstadt, Germany and \\ ExtreMe Matter Institute EMMI, GSI Helmholtzzentrum \\ für Schwerionenforschung, 64291 Darmstadt, Germany \\ E. Hiyama \\ RIKEN Nishina Center, RIKEN, Saitama 351-0198, Japan \\ (Dated: October 21, 2019)
}

\begin{abstract}
We calculate the universal spectrum of trimer and tetramer states in heteronuclear mixtures of ultracold atoms with different masses in the vicinity of the heavy-light dimer threshold. To extract the energies, we solve the three- and four-body problem for simple two- and three-body potentials tuned to the universal region using the Gaussian expansion method. We focus on the case of one light particle of mass $m$ and two or three heavy bosons of mass $M$ with resonant heavy-light interactions. We find that trimer and tetramer cross into the heavy-light dimer threshold at almost the same point and that as the mass ratio $M / \mathrm{m}$ decreases, the distance between the thresholds for trimer and tetramer states becomes smaller. We also comment on the possibility of observing exotic three-body states consisting of a dimer and two atoms in this region and compare with previous work.
\end{abstract}

PACS numbers: 31.15.ac, 03.65.Ge, 67.85.-d, 21.45.-v

\footnotetext{
* schmickler@theorie.ikp.physik.tu-darmstadt.de
} 


\section{INTRODUCTION}

Particles with resonant short-range interactions display the Efimov effect [1] and related universal phenomena associated with a discrete scaling symmetry [2-4]. In particular, Efimov showed that three identical bosons form infinitely many trimer bound states with an accumulation point at the scattering threshold when the $s$-wave scattering length $a$ is tuned to the unitary limit $1 / a=0$ :

$$
E_{3}^{(n)}=-\left(e^{-2 \pi / s_{0}}\right)^{n} \frac{\hbar^{2} \kappa_{*}^{2}}{m}
$$

where $m$ is the mass of the particles, $s_{0}=1.00624 \ldots$ is a transcendental number, and $\kappa_{*}$ is the binding wavenumber of the Efimov state labeled by $n=0$. The geometric spectrum in (1) is the signature of a discrete scaling symmetry with scaling factor $e^{\pi / s_{0}} \approx 22.7$. For a finite scattering length larger than the range of the interaction, the universal properties persist but there is only a finite number of Efimov states. The full structure of the trimer spectrum as a function of the inverse scattering length $1 / a$ is conveniently summarized in the so-called Efimov plot illustrated in Fig. 1 . This plot shows the scattering length dependence of the bound states in the $a^{-1}-K$ plane, where

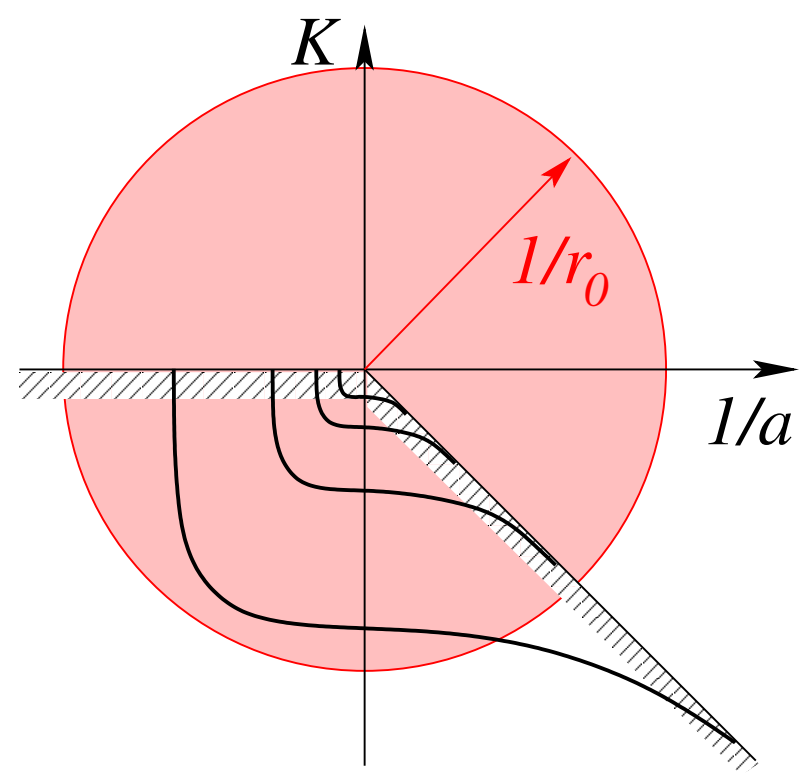

FIG. 1. The $a^{-1}-K$ plane for the 3-body problem. Scattering thresholds are indicated by the cross-hatching while the universal window is given by the circle of radius $1 / r_{0}$. A few of the infinitely many branches of Efimov states are shown by the solid lines.

$K=\operatorname{sgn}(E) \sqrt{m E}$ is a momentum variable that corresponds to the binding momentum for bound states with $E<0$. The threshold for scattering states is indicated by the hatched area. The Efimov trimers are represented by the solid lines below the threshold. There are infinitely many branches of Efimov trimers, but only a few are shown. In the unitary limit $1 / a=0$, infinitely many trimer states accumulate at threshold. When the radial variable $\sqrt{K^{2}+1 / a^{2}}$ is of the order of the inverse interaction range $1 / r_{0}$ or larger, universality breaks down and the states become sensitive to the details of the interaction at short distances. 
Ultracold atoms are an ideal tool to study such phenomena since the scattering length can be tuned experimentally to be large compared to the range of the interaction using Feshbach resonances. Efimov trimers can be observed in ultracold atomic gases via their signature in three-body recombination rates [5-9]. Using this method, Kraemer et al. provided the first evidence for Efimov trimers in an ultracold gas of ${ }^{133} \mathrm{Cs}$ atoms by observing the resonant enhancement of atom losses through three-body recombination caused by the trimers [10]. Efimov states have been observed in a variety of other atomic species since then [4, 11].

The scaling factor $e^{\pi / s_{0}}$ can be significantly reduced in heteronuclear systems with different mass particles which makes it easier to observe multiple states. In this paper, we focus on heteronuclear systems with two species of atoms where only the interspecies scattering length is large. For comparable masses the scaling factor is quite large in this case $\left(e^{\pi / s_{0}} \approx 1986.1\right.$ for equal masses) as we now have only two resonant interactions out of three. However, in the case of two heavy bosonic atoms of mass $M$ (denoted $H$ ) and one light atom of mass $m<M$ (denoted $L$ ), this factor can become significantly smaller than the value 22.7 for identical bosons [2, 12,14]. Relaxation and recombination losses near an interspecies resonance have recently been investigated in mixtures of Cesium and Lithium atoms and three consecutive Cs-Cs-Li Efimov resonances were observed in agreement with the predicted scaling relations [15-19]. The effect of the Cs-Cs scattering length on the Efimov resonances was investigated in Refs. [20-22] [1]

Connected to the Efimov states are universal bound states in the four- and higher-body sector. Although there is no accumulation of bound states at threshold, there are universal four- and higher-body states attached to each Efimov trimer. The binding energies of these higher-body states are uniquely determined by $a$ and $\kappa_{*}$ and no higher-body parameters are required [23]. In experiments with ultracold Cesium atoms universal states up to $N=5$ have been observed [24]. For identical bosons, exactly two tetramer states are attached to each trimer state [25-28]. This pattern appears to hold also for the higher-body states and has been calculated explicitly up to $N=6$ [29, 30]. The ground states have been calculated up to $N=16$ [31- -33].

The demonstration of the existence of the higher-body universal states in experiments with mixtures represents a frontier in the physics of ultracold atoms. Only recently a loss feature attributed to a $\mathrm{Na}_{2} \mathrm{Rb}_{2}$ state has been seen in an experiment involving $\mathrm{NaRb}$ Feshbach molecules [34]. Focusing on four-body states, the $H_{3} L$ states are very promising due to the favorable scaling factor. However, the relation of the higher-body energies to the trimer energies is not governed by the same universal numbers and even the number of higher-body states attached to the trimers will very likely depend on the mass ratio. The signatures of Efimov physics in heteronuclear four-body systems were first investigated in Ref. [35]. A detailed study of the four-body states in a mixture of Cesium and Lithium atoms in the vicinity of the unitary limit and the three-atom threshold was recently performed by Blume and Yan [36]. For large enough mass ratios $(M / m \geq 16)$ they found two universal four-body states in the unitary limit. They also found an excited four-body state for negative values of the scattering length for all mass ratios they investigated ( $8 \leq M / m \leq 50$ ). For the mass ratio $M / m=133 / 6$ they showed that the excited tetramer becomes unbound at a certain positive value of the scattering length, leading them to the conjecture that the scattering length at which the excited tetramer becomes unbound depends on the mass ratio.

In this paper, we build upon the investigation by Blume and Yan and extend it in several ways. We extend their calculation to positive scattering lengths further away from the unitary limit. In particular, we investigate the mass dependence of the spectrum and discuss the number of universal tetramer states as function of the mass ratio. One of our key findings is that the structure of the spectrum changes as the mass ratio $M / m$ decreases. In particular, the distance between the

\footnotetext{
${ }^{1}$ Note that there should also be Li-Li-Cs Efimov resonances, but in this case the scaling factor is extremely large.
} 
thresholds for trimer and tetramer states gets smaller until they almost coincide for $M / m \approx 1$. Preliminary studies of this aspect were presented in Ref. [38]. Moreover, we summarize our insights in a multi-dimensional Efimov plot which shows $K$ as a function of $1 / a$ and the ratio of the heavy and light masses $M / m$.

A particularly interesting aspect of the $H_{3} L$ system is the prospect of observing effective Efimov states of a $H L$ dimer and two heavy atoms $H$ which are denoted $H_{2}(H L)$ in the following. Such an effective Efimov effect must exist close to the crossing point of the $H_{2} L$ trimer and the $H L$ dimer threshold. This requires precise studies of the bound state spectrum near the $H L$ dimer threshold. Assuming that only the $H L$ dimer and the two $H$ atoms interact, one would expect a scaling factor that is larger than 1986, which is the limiting value for $M / m \rightarrow \infty$ [2]. For a Cesium-Lithium mixture, one would expect a scaling factor of about 2500.

Using the Born-Oppenheimer approximation, Wang and collaborators [35] found evidence for the existence of two such states for the mass ratio $M / m=50$ with a scaling factor of approximately 20. They explain the small scaling factor with an effective interaction between the $H$ particles that is induced by the exchange of the $L$ particle. In this paper, we will address this puzzle by investigating the effective $H_{2}(H L)$ Efimov states for different mass ratios $\mathrm{M} / \mathrm{m}$ using explicit four-body calculations as well as using an effective field theory for heteronuclear threebody mixtures [37].

\section{METHOD}

To obtain the energies of the $H_{2} L$ trimer and $H_{3} L$ tetramer systems, we employ the Gaussian expansion method (GEM) [39]. The method was successfully applied to various types of three- , four- and five-body hypernuclear systems [40-45] and trimer and tetramer systems of ${ }^{4} \mathrm{He}$ [46-48].

We use Jacobian coordinate sets for trimer and tetramer systems as shown in Fig. 2, The Hamiltonians for the three and four-body systems are described as

$$
\begin{aligned}
& H=T+\sum_{i=1}^{2} V_{A_{i} A_{3}}+V_{A_{1} A_{2} A_{3}} \\
& H=T+\sum_{i=1}^{3} V_{A_{i} A_{4}}+\sum_{i<j}^{3} V_{A_{i} A_{j} A_{4}}
\end{aligned}
$$

respectively, where $T$ is the kinetic-energy operator, the $V_{A_{i} A_{3}}$ and $V_{A_{i} A_{4}}$ are two-body potentials and $V_{A_{1} A_{2} A_{3}}$ and $V_{A_{i} A_{j} A_{4}}$ are three-body potentials, which are the same as in Ref. [36]. The details are given below. Here it should be noted that we switch off the two-body interaction between the heavy bosonic atoms.

The energies are obtained by solving the trimer and tetramer Schrödinger equations given by

$$
\begin{aligned}
(H-E) \Psi_{\text {trimer }} & =0, \\
(H-E) \Psi_{\text {tetramer }} & =0 .
\end{aligned}
$$

The wave functions of three-body and four-body systems are described as a sum of amplitudes for the rearrangement channels ( $c=1,2$ for the trimer, $c=1, \ldots, 4$ for the tetramer) illustrated in 
(a)

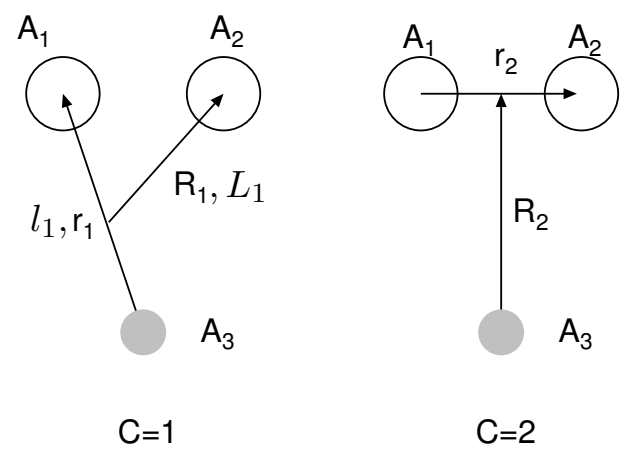

(b)
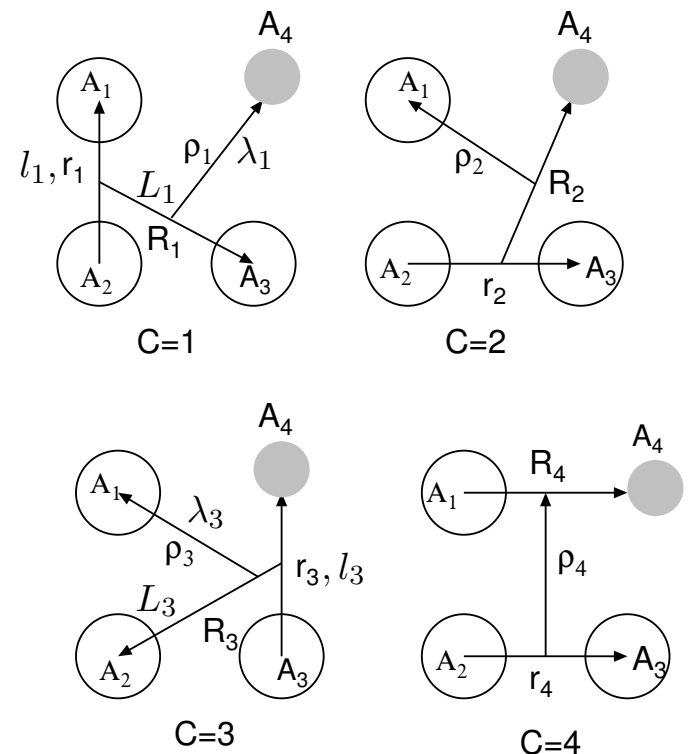

FIG. 2. Jacobian coordinates for trimer (a) and tetramer (b) systems. In (a) $A_{1}$ and $A_{2}$ are heavy bosonic atoms and $A_{3}$ is a light atom. In (b) $A_{1}, A_{2}$, and $A_{3}$ are heavy bosonic atoms and $A_{4}$ is a light atom. The two bosonic atoms in the trimer and the three bosonic atoms in the tetramer are symmetrized.

Fig. 2 as follows:

$$
\begin{aligned}
\Psi_{J M}^{\text {trimer }}= & \sum_{c=1}^{2} \sum_{n_{c}, N_{c}} \sum_{\ell_{c}, L_{c}} C_{n_{c} \ell_{c} N_{c} L_{c}}^{(c)} \\
& \times\left[\phi_{n_{c} \ell_{c}}^{(c)}\left(\mathbf{r}_{c}\right) \psi_{N_{c} L_{c}}^{(c)}\left(\mathbf{R}_{c}\right)\right]_{J M} \\
\Psi_{J M}^{\mathrm{tetramer}}= & \sum_{c=1}^{4} \sum_{n_{c}, N_{c}, \nu_{c} \ell_{c}, L_{c}, \lambda_{c}} \sum_{n_{c} \ell_{c} N_{c} L_{c} \nu_{c} \lambda_{c}}^{(c)} \\
& \times\left[\left[\phi_{n_{c} \ell_{c}}^{(c)}\left(\mathbf{r}_{c}\right) \psi_{N_{c} L_{c}}^{(c)}\left(\mathbf{R}_{c}\right)\right]_{I} \xi_{\nu_{c} \lambda_{c}}^{(c)}\left(\boldsymbol{\rho}_{c}\right)\right]_{J M} .
\end{aligned}
$$

Here, the two (three) bosonic atoms in the trimer (tetramer) must be symmetrized. For this purpose, we take the angular momenta $\ell$ to be even numbers in the corresponding rearrangement channels. We take the functional form of $\phi_{n \ell m}(\mathbf{r}), \psi_{N L M}(\mathbf{R})$, and $\xi_{\nu \lambda \mu}(\boldsymbol{\rho})$ as

$$
\begin{aligned}
\phi_{n l m}(\mathbf{r}) & =\phi_{n l}^{\mathrm{G}}(r) Y_{l m}(\widehat{\mathbf{r}}), & \phi_{n l}^{\mathrm{G}}(r) & =N_{n l} r^{l} e^{-\left(r / r_{n}\right)^{2}}, \\
\psi_{N L M}(\mathbf{R}) & =\psi_{N L}^{\mathrm{G}}(R) Y_{L M}(\widehat{\mathbf{R}}), & \psi_{N L}^{\mathrm{G}}(R) & =N_{N L} R^{L} e^{-\left(R / R_{N}\right)^{2}}, \\
\xi_{\nu \lambda \mu}(\boldsymbol{\rho}) & =\xi_{\nu \lambda \mu}^{\mathrm{G}}(\boldsymbol{\rho}) Y_{\lambda \mu}(\widehat{\boldsymbol{\rho}}), & \xi_{\nu \lambda}^{\mathrm{G}}(\rho) & =N_{\nu \lambda} \rho^{\lambda} e^{-\left(\rho / \rho_{\nu}\right)^{2}},
\end{aligned}
$$

where $N_{n l}, N_{N L}, N_{\nu \lambda}$ denote the normalization constants. The Gaussian range parameters are 
chosen according to geometrical progression:

$$
\begin{array}{lll}
r_{n}=r_{\min } a^{n-1}, & a=\left(\frac{r_{\max }}{r_{\min }}\right)^{\frac{1}{n_{\max }-1}} & \left(n=1 \sim n_{\max }\right), \\
R_{n}=R_{\min } A^{N-1}, & A=\left(\frac{R_{\max }}{R_{\min }}\right)^{\frac{1}{N_{\max }-1}} & \left(N=1 \sim N_{\max }\right), \\
\rho_{n}=\rho_{\min } \alpha^{\nu-1}, & \alpha=\left(\frac{\rho_{\max }}{\rho_{\min }}\right)^{\frac{1}{\nu_{\max }-1}} & \left(\nu=1 \sim \nu_{\max }\right) .
\end{array}
$$

The Gaussian ranges are very suitable for accurately describing both the short-range correlations and the long-range tail in the asymptotic region of few-body wave functions. The Gaussian shape of basis functions makes the calculation of matrix elements simple even between basis functions of different channels.

\begin{tabular}{|c|c|c|c|c|c|c|}
\hline$c l_{c} L_{c}$ & $n_{\max }$ & $r_{\text {min }}$ & $r_{\max }$ & $N_{\max }$ & $R_{\text {min }}$ & $R_{\max }$ \\
\hline $\begin{array}{lll}1 & 0 & 0\end{array}$ & 23 & 0.028 & 15 & 20 & 0.038 & 65 \\
\hline 122 & 21 & 0.180 & 27 & 19 & 0.228 & 20 \\
\hline $\begin{array}{lll}2 & 0 & 0\end{array}$ & 19 & 0.110 & 26 & 21 & 0.085 & 29 \\
\hline
\end{tabular}

TABLE I. Typical three-body angular momentum space and Gaussian range parameters employed for the trimer system. The channel numbers refer to Fig. 2 .

The eigenenergies $E$ in Eq. (3) and the coefficients $C$ in Eq. (4) are then determined by the Raleigh-Ritz variational method.

In Tables $\Pi$ and $\Pi$, typical parameters of the basis functions for the trimer and tetramer are listed.

TABLE II. Typical four-body angular momentum space and Gaussian range parameters employed for the tetramer system. The channel numbers refer to Fig. 2 .

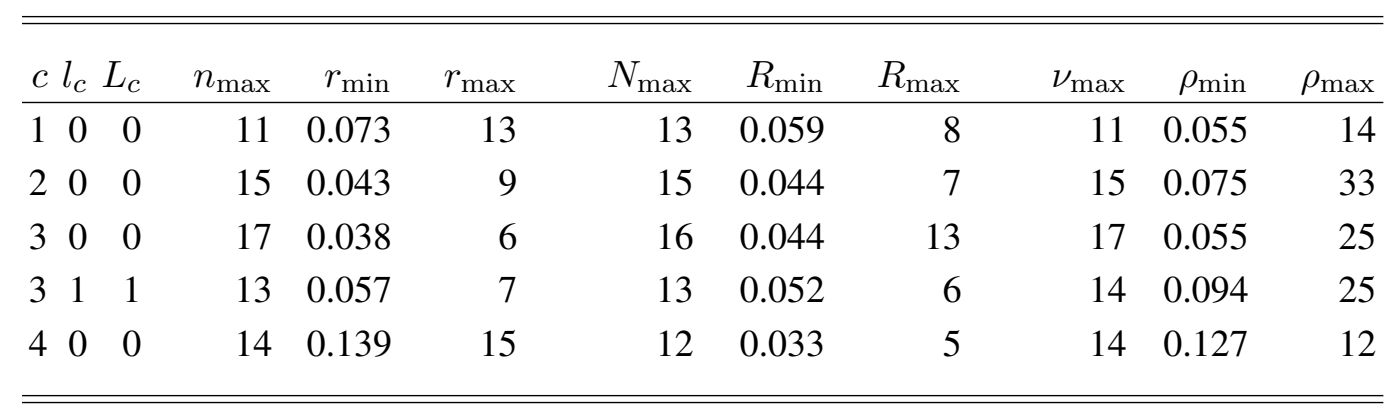

The potential and kinetic energy terms are expanded in the truncated basis as shown in Eq. (4). 
Because the states are not orthogonal this transforms the Schrödinger equation

$$
\sum_{c} \sum_{n_{c} l_{c} N_{c} L_{c}}\left\langle\left[\phi_{n_{c^{\prime}} \ell_{c^{\prime}}}^{\left(c^{\prime}\right)}\left(\mathbf{r}_{c^{\prime}}\right) \psi_{N_{c^{\prime}} L_{c^{\prime}}}^{\left(c^{\prime}\right)}\left(\mathbf{R}_{c^{\prime}}\right)\right]_{J M}|H-E| C_{n_{c} l_{c} N_{c} L_{c}}^{(c)}\left[\phi_{n_{c} \ell_{c}}^{(c)}\left(\mathbf{r}_{c}\right) \psi_{N_{c} L_{c}}^{(c)}\left(\mathbf{R}_{c}\right)\right]_{J M}\right\rangle=0
$$

into a general eigenvalue problem

$$
\sum_{\tilde{n}}\left(H_{\tilde{n}^{\prime} \tilde{n}}-E N_{\tilde{n}^{\prime} \tilde{n}}\right) C_{\tilde{n}}=0
$$

where $N_{\tilde{n}^{\prime} \tilde{n}}$ is a normalization matrix and $\tilde{n}$ is a shorthand notation for all the indices that are summed over, i.e. $\tilde{n} \triangleq\left(c, n_{c}, l_{c}, N_{c}, L_{c}\right)$ in the three-body case. The matrix elements can be calculated analytically for some potentials and the equation can be solved using standard linear algebra methods.

Care has to be taken to avoid overcompleteness of the basis sets. We meticulously checked the convergence behavior and the behavior of the binding energies under slight variations of the potential depth to discard overcomplete basis sets.

\section{INTERACTION}

Since we want to study universal effects, i.e. effects that are independent of the exact shape of the potential and only depend on the long-range behavior of the potential, it is beneficial to use simple potentials. We have chosen Gaussian potentials, because their matrix elements can be calculated analytically in the Gaussian Expansion Method (GEM). There are many prior calculations with Gaussian potentials [36, 49], so we can easily validate our method.

We use the same potentials as in Ref. [36]. The two-body potential is given by

$$
V_{A_{i} A_{N}}=v_{0} e^{-\frac{r_{i N}^{2}}{2 r_{0}^{2}}}
$$

and the three-body potential is

$$
V_{A_{i} A_{j} A_{N}}=w_{0} e^{-\frac{r_{i j}^{2}+r_{i N}^{2}+r_{j N}^{2}}{16 r_{0}^{2}}}
$$

where $r_{i j}=r_{i}-r_{j}$, and $r_{i}$ is the position of atom $i$. The interaction takes place only between different atoms, where the $N$ th atom is the special one (type $L$ ), and all other atoms are identical bosons (type $H$ ). The interaction between identical bosons is neglected, because it is assumed to be very weak compared to the resonant $H L$ interaction. This is reflected in the summation in Eq. (2).

Gaussian potentials that are tuned to the universal regime can be understood as regularized contact terms in an EFT expansion [49], so our results are valid only in the energy regime of this implicit EFT expansion. This requires that all calculated energies are small compared to the natural energy scale set by the window of universality shown in Fig. 1. The natural energy scale connected to the potential 13,14 is

$$
E_{s}=\frac{\hbar^{2}}{2 \mu r_{0}^{2}}, \quad \text { with } \quad \mu=\frac{m M}{m+M}
$$




\begin{tabular}{lllll}
\multicolumn{2}{c}{$w_{0} / E_{s}$} & \multicolumn{2}{c}{$\sqrt{E_{3}^{0} / E_{s}}$} & \multicolumn{2}{c}{$\sqrt{E_{3}^{0} / E_{3}^{1}}$} \\
& our result & {$[36]$} & our result & {$[36]$} \\
\hline 0.00 & 0.314347 & 0.314348 & 3.9341 & 3.9326 \\
0.16 & 0.188463 & 0.188467 & 4.1225 & 4.1205 \\
2.56 & 0.031410 & 0.031417 & 4.8955 & 4.8968 \\
9.60 & 0.025999 & 0.026004 & 4.9023 & 4.9025
\end{tabular}

TABLE III. Comparison of our benchmarking results with [36] for the mass ratio $M / m=133 / 6$. The numbers are taken from Fig. 2 of the supplement and were provided to us by D. Blume [50]. The first column gives the strength of the three-body force in units of the natural energy scale $E_{s}$ as defined in Eq. (15). The following columns give the ratios of the trimer ground state and $E_{s}$ and the ratio of the lowest two trimer states, respectively.

\begin{tabular}{lllll}
\multicolumn{2}{c}{$w_{0} / E_{s}$} & \multicolumn{2}{c}{$\sqrt{E_{4}^{0,0} / E_{3}^{0}}$} & \multicolumn{2}{c}{$\sqrt{E_{4}^{0,1} / E_{3}^{0}}$} \\
& our result & {$[36]$} & our result & {$[36]$} \\
\hline 0.00 & 1.89891 & 1.89890 & & \\
2.56 & 1.51452 & 1.51425 & 1.0119 & 1.0116 \\
9.60 & 1.51121 & 1.51119 & 1.0110 & 1.0106 \\
\hline
\end{tabular}

TABLE IV. Comparison of our benchmarking results with [36] for the mass ratio $M / m=133 / 6$. The numbers are taken from Fig. 3 of the supplement and were provided to us by D. Blume [50]. The first column gives the strength of the three-body force in units of the natural energy scale $E_{s}$ as defined in Eq. (15). The following columns show the ratio of the tetramer ground state and the trimer ground state and the ratio of the tetramer excited state and the trimer ground state, respectively.

the reduced mass of the interacting dimer $(H L)$. The two-body potential $(13)$ is attractive, whereas the three-body potential (14) is chosen to be repulsive such that the calculated binding energies are in the window of universality, i.e. $B \ll E_{s}$. The three-body force parameter, $w_{0}$, is kept constant throughout the calculations presented here, except where noted otherwise.

The units are chosen so that the calculations are numerically well-behaved, and the results are presented in terms of $E_{s}$ and $a$, the $H L$ s-wave scattering length. We vary $v_{0}$ to reproduce different scattering lengths $a$ as $v_{0}$ is roughly linearly proportional to $1 / a$. The length scale $r_{0}$ is then essentially a free parameter that sets the scale for $a$. Its numerical value is set to 0.04 throughout our calculations.

\section{BENCHMARKING}

In order to benchmark our method, we calculate the trimer and tetramer energies in the vicinity of the unitary limit and compare with the results for the mass ratio $\mathrm{M} / \mathrm{m}=133 / 6 \mathrm{ob}$ tained by Blume and Yan [36]. Our calculations are carried out very close to the unitary limit at $|a| / r_{0}=10^{10}$. The corresponding results for the trimer energies are given in Table III, while the corresponding tetramer energies are given in Table IV. For the different strengths of the three-body force $w_{0}$, we find agreement with Ref. [36] to three digits or better, which gives us confidence in our method.

However, the time spent optimizing the basis function parameters for the actual calculations 
discussed in Sec. VI was about 20 times longer than for the benchmark calculations discussed above. Moreover, the basis size used for the actual calculations was about twice as large as for the benchmark calculations. This ensures that our results in Sec. $\mathrm{VI}$ are converged to about 8 digits corresponding to a precision of $10^{-8} \sqrt{E_{s}}$.

The energies are less sensitive to non-universal aspects of the finite-range model potentials for larger values of $w_{0}$ [36]. As a consequence, we use $w_{0} / E_{s} \approx 9.6$ in our calculations of the $H_{2} L$ and $H_{3} L$ systems below. This ensures absolute energies are small enough that range effects do not play a role and the results are close to the universal limit of zero-range interactions. In practice, we have ensured that all calculated energies satisfy $E \leq 0.13 E_{s}$.

Since the Gaussian Expansion Method as employed here uses a truncated sum over rearrangement channels and relative angular momenta to represent the wave function (cf. Eq. (4)), contributions from higher relative angular momenta are neglected. We included as many configurations as possible without encountering numerical instabilities. In addition, the states are extremely shallow in the region where very high precision is required, such that higher relative angular momenta are strongly suppressed. The highest angular momentum quantum numbers that are included in our four-body calculations are $L_{3}=1, \lambda_{3}=1$. Including the corresponding $L_{3}=1, \lambda_{3}=1$ basis functions of the rearrangement channel 3 (cf. Fig. 2), which contributes the most, leads to a correction of only $10^{-7} \sqrt{E_{s}}$ near the threshold. This gives us confidence that including even higher relative angular momenta would not lead to significant contributions.

\section{EFIMOV PLOT FOR HETERONUCLEAR SYSTEMS}

In this work, we focus on three-body systems of one light particle $L$ of mass $m$ and two heavy bosons $H$ of mass $M$ and four-body systems of three heavy bosons $H$ and one light particle $L$. In order to establish the qualitative behavior of trimer and tetramer states as a function of the mass ratio $M / m$, we have calculated the tetramer and trimer energies for the cases $(M=133, m=6)$, $(M=87, m=7)$, and $(M=7, m=6)$, which correspond to possible mixtures of ultracold atoms, as a function of $r_{0} / a$ where $r_{0}$ is the interaction range of the effective potentials considered (cf. Eqs. (13, 14)). Thus the scale $E_{s}$ defined in Eq. (15) is the natural energy scale of the problem, and all energies are quoted in units of $E_{s}$. To embed our findings in a larger context, we show in Fig. 3 an Efimov plot for $H_{2} L$ trimer and $H_{3} L$ tetramer states for the mass ratios $M / m=133 / 6$ and $M / m=7 / 6$. All states shown are well within the universal window indicated by the shaded area in Fig. 1. The results for $M / m=87 / 7$ are not plotted because they would overlap with the results for $M / m=133 / 6$. To increase visibility of the features of the plot, the axes are rescaled by taking the fourth root. The variables $H$ and $\xi$ are introduced as $r_{0} / a=H \cos \xi$ and $-\sqrt{|E| / E_{s}}=H \sin \xi$ (cf. Ref. [25]). ${ }^{2}$ We only show the lowest trimer because we focus on the tetramer states attached to each trimer for two different mass ratios. We could confirm that, as already noted by Blume at al. [36], the excited tetramer does exist in the unitary limit for $M / m=133 / 6$ and does not for $M / m=7 / 6$. They reported that for mass ratios less than 16, namely 12 and 8, they could only find an excited tetramer away from the unitary limit on the negative scattering length side. For $M / m=7 / 6$ we did not find any excited tetramer, not even on the negative scattering length side. This indicates that the value of $1 / a$ where the excited tetramer crosses into the trimer threshold recedes from a point on the positive scattering length side of the Efimov plot for large mass ratios to points on the negative scattering length side for smaller mass

\footnotetext{
${ }^{2}$ Due to the rescaling of $H$ to $H^{1 / 4}$ small deviations from the linearity of the dimer threshold are strongly magnified. Therefore we plot the energy differences to the $H L-H-H$ dimer-atom-atom threshold on the right half plane and include an idealized dimer-atom-atom threshold.
} 
ratios until it ceases to exist altogether. However, to prove this hypothesis, more data points are necessary. In a very recent study [34], the ${ }^{23} \mathrm{Na}-{ }^{87} \mathrm{Rb}$-system has been calculated. In this case with a mass ratio of about 3.8 no excited state was found, which agrees with our findings.

Comparing the curves for the two mass ratios, one can see that the $M / m=7 / 6$ mixture has much shallower bound states, approaching the dimer threshold with an extremely small slope. This holds true even when the three-body potential strength is adjusted such that the trimer vanishes at the same value for $r_{0} / a$ for both mixtures. In addition, in both cases the tetramer and the trimer seem to vanish at the same point. This result was also corroborated in [34] for the $\mathrm{NaRb}_{2}$ trimer and the $\mathrm{NaRb}_{3}$ tetramer. In the next section, we show our results for this point with much higher resolution and discuss it in more detail.

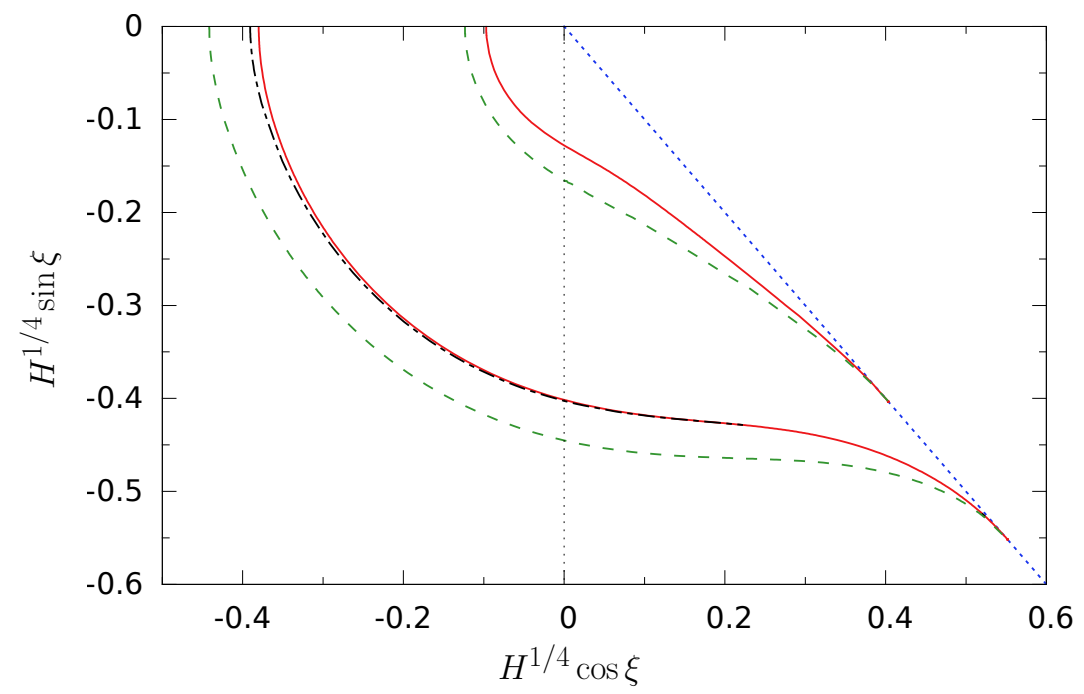

FIG. 3. Efimov plot for $H_{2} L$ trimers and $H_{3} L$ tetramers in a ${ }^{133} \mathrm{Cs}^{6}{ }^{6} \mathrm{Li}$ mixture (lower group of lines) and a ${ }^{7} \mathrm{Li}-{ }^{6} \mathrm{Li}$ mixture (upper group of lines). The blue dotted line marks the $H L$ dimer + two $H$ atom threshold, the red solid lines the $H_{2} L$ trimer $+H$ atom threshold, the dashed green lines the $H_{3} L$ tetramer ground states, and the dot-dashed black line the excited state of the tetramer. To increase visibility of the features of the plot, the axes are rescaled by taking the fourth root. To this end, $H$ and $\xi$ are introduced as $r_{0} / a=H \cos \xi$ and $-\sqrt{|E| / E_{s}}=H \sin \xi$ (cf. Ref. [25]).

\section{TETRAMER AND TRIMER CLOSE TO THE DIMER THRESHOLD}

Next we focus on the tetramer spectrum close to the atom-dimer threshold in the region where the trimer disappears. This region has not been studied in a full four-body approach for heteronuclear systems. The corresponding results are shown in Figs. 4 and 5 , respectively. In all three cases $(M=133, m=6),(M=87, m=7)$, and $(M=7, m=6)$, the tetramer disappears through the dimer threshold after the trimer has already disappeared. However, the points in $r_{0} / a$ where the trimer and tetramer disappear approach each other as the mass ratio $M / m$ is decreased and the two states almost disappear at the same point in the $(M=7, m=6)$ case. This is a qualitatively 


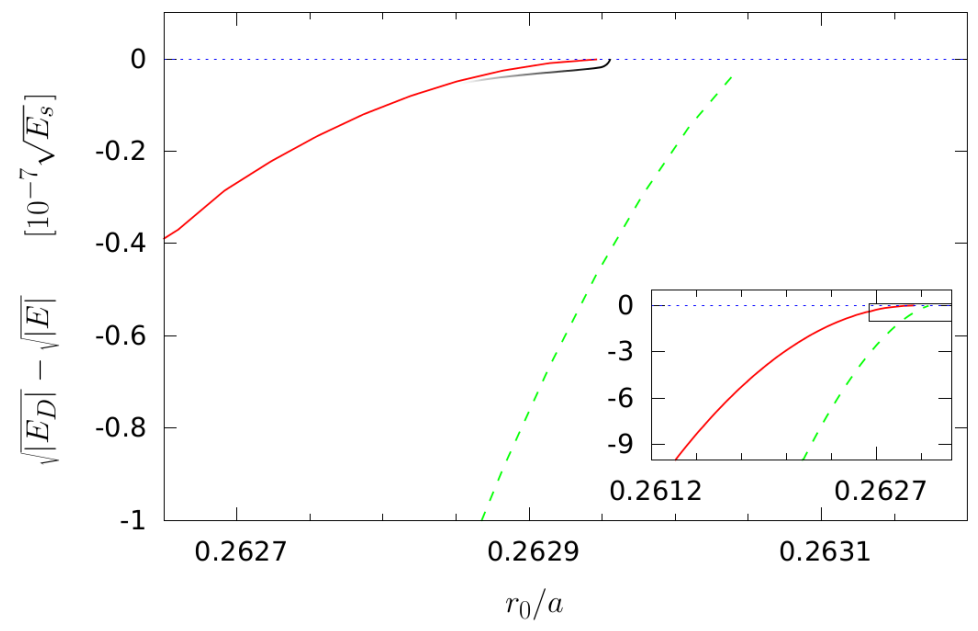

FIG. 4. Binding energies of tetramer (dashed line) and trimer (solid line) of the 133-133-6 system in the region close to the $H L$ dimer threshold (dotted line). $E_{D}$ is the $H L$ dimer energy and $E_{s}$ is the natural energy scale defined in Eq. (15). The inset shows a larger region of $r_{0} / a$. We included our estimate (solid black line) of where the next excited Efimov state should appear if the scaling factor were 20 (see discussion in Sec. VII C). We did not find evidence for such a state.

new behavior that is not seen in systems with identical bosons. It might suggest that for equal masses $M=m$, the two states disappear at the same value of $r_{0} / a$. We have further investigated this below. If the mass ratio $\mathrm{M} / \mathrm{m}$ is even further decreased, we enter the regime of an inverted mass ratio where the scaling factor grows rapidly. In the current study we have not investigated this region.

To quantify the behavior of the threshold crossings, we have extracted the difference $c_{d}=$ $c_{4}-c_{3}$ between the points $c_{4}$, denoting the value of $r_{0} / a$ where the $H_{3} L$ tetramer vanishes into the $\mathrm{HL}+\mathrm{H}+\mathrm{H}$-threshold, and $c_{3}$, the point where the $\mathrm{H}_{2} \mathrm{~L}$ trimer vanishes into the $H \mathrm{~L}+H$-threshold, from Figs. 4 and 5 . We show this coefficient as a function of the mass ratio $M / m$ in Fig. 6 , Our results for the mass ratios $M / m=7 / 6,87 / 7,133 / 6$ are shown by the circle, square, and triangle, respectively. The errors in the extracted values are relatively large, but our calculations indicate that the relation is not linear.

In order to understand this pattern, we have performed effective field theory calculations for effective three-body systems of an $H L$ dimer of mass $M+m$ and two $H$ bosons of mass $M$, where only the dimer and the bosons interact. Using the effective field theory formalism for unequal mass particles with resonant interaction discussed in Ref. [37], we can calculate the coefficient $c_{d}$ up to one free parameter, namely the effective three-body parameter of the $H L-H-H$ system. (See Appendix A for a brief discussion of this framework.) Therefore we can fit the effective threebody parameter either to the point $M / m=87 / 7$ (dashed line) or $M / m=133 / 6$ (solid line). In each case the point $M / m=7 / 6$ is reproduced within error bands. A fit that reproduces all three points is not possible. This shows the limitations of the effective three-body calculation which is strictly valid only near $c_{d} \approx 0$. The effective three-body calculation shows that $c_{d}$ vanishes for $M / m \rightarrow 0$, and is very small but finite at $M=m$. We emphasize that this effective three-body 


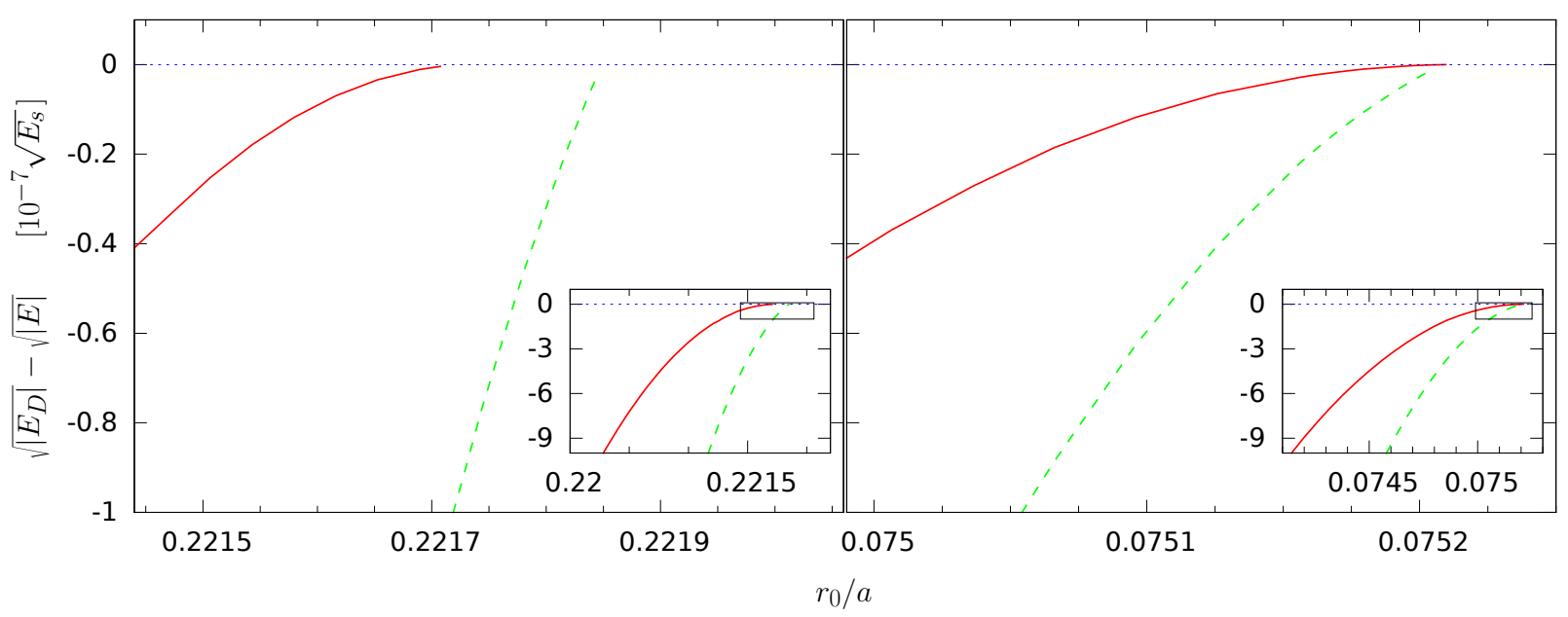

FIG. 5. Binding energies of tetramer (dashed line) and trimer (solid line) of the 87-87-7 system (left panel) and of the 7-7-6 system (right panel) in the region close to the $H L$ dimer threshold (dotted line). $E_{D}$ is the $H L$ dimer energy and $E_{s}$ is the natural energy scale defined in Eq. (15). The inset shows a larger region of $r_{0} / a$.

approach is not exact and should only be valid near the point where the trimer vanishes through the $H L$ dimer threshold. An estimate of the $H L-H$ scattering length indicates that its value is at least two orders of magnitude larger for the case $M / m=7 / 6$ compared to the other two mass ratios. Thus we expect the effective three-body picture to work best for this case.

The tension between effective three-body and the GEM calculations could be due to two potential problems. First, the GEM calculations could be not as accurate as we estimate, possibly due to neglected higher partial wave contributions or hard to detect numerical pathologies. Second, some essential aspect of the system might not be present in the effective three-body description, which might also explain why different approximation schemes yield different results as discussed in the next section.

\section{DIMER-ATOM-ATOM EFIMOV STATES}

To discuss the effective Efimov states that were already mentioned in the introduction, three aspects are important. To begin with, the region where they can occur has to be established. For discussions on whether or not they can be seen in numerical calculations or even in experiments, an important issue is the predicted scaling factor. We will briefly present two competing approximation schemes that lead to very different predictions. To conclude, we will comment on the current state of the evidence for the effective Efimov states. 


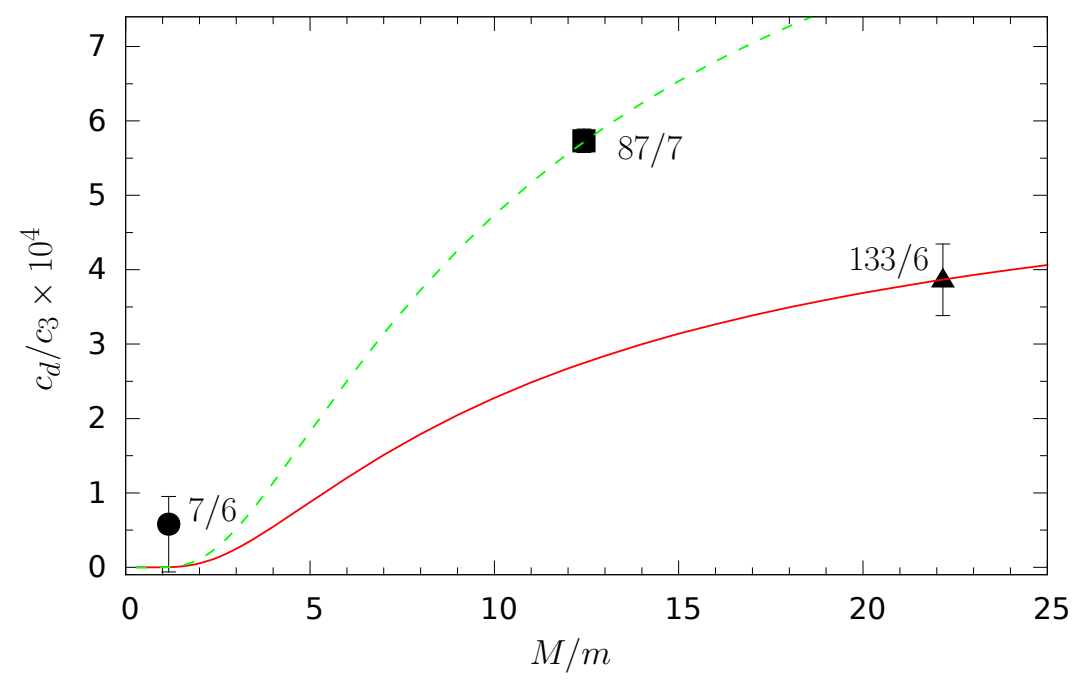

FIG. 6. Difference between trimer and tetramer thresholds in units of $r_{0} / a$ as a function of the mass ratio $M / m=7 / 6,87 / 7,133 / 6$ (circle, square, triangle). The dashed (solid) line shows effective field theory calculations for an effective three-body system of an $H L$ dimer and two heavy bosons $H$ of mass $M$ fitted to reproduce the point $M / m=87 / 7(M / m=133 / 6)$.

\section{A. Motivation and Region of Possible Occurrence}

Near the dimer threshold, both trimer and tetramer become very weakly bound in comparison to the dimer. For this reason it is expected that at a certain point the system can be treated as an effective three-body problem. At the point were the trimer becomes unbound, the dimer-atom interaction is resonant, leading to the occurrence of the Efimov effect in the effective three-body system. Therefore, the effective Efimov effect is expected to arise in a small region around the point where the trimer crosses the dimer threshold. This region is bounded by the tetramer ground state from below. As we have shown in the previous section, this region (the size of which can be expressed by $c_{d} / c_{3}$ ) proved to be extremely small in our calculations. The effective Efimov effect has already been studied in systems of four identical bosons by Deltuva [51] and in heteronuclear systems by Wang et al. [35].

For four identical bosons, Deltuva found $c_{d} / c_{3}<1.2 \times 10^{-6}$, which compares well to our value for ${ }^{7} \mathrm{Li} /{ }^{6} \mathrm{Li}, c_{d} / c_{3}=5.8 \times 10^{-5}$. For equal masses, our effective three-body calculations yielded values between $10^{-8}$ and $10^{-7}$, depending on the three-body parameter (compare Fig. 6 and the discussion in the previous section). Assuming that the system is away from the unitary limit and close to the dimer threshold the dimer-atom scattering length is much larger than the scattering length between identical bosons, which means that the system studied in [51] is comparable to our calculations. Deltuva also did effective three-body dimer-atom-atom calculations and showed that very close to the dimer-atom-atom threshold this approximation is valid. His estimated scaling factor for the effective Efimov states is $5 \times 10^{5}$, which agrees with [2] and our discussion in the next section. 
In a paper from 2012, Wang et al. [35] used the Born-Oppenheimer approximation in heteronuclear systems with very high mass ratios $(M / m=30$ and 50) to calculate recombination features. They calculated trimer $\left(\mathrm{H}_{2} \mathrm{~L}\right)$, tetramer $\left(\mathrm{H}_{3} \mathrm{~L}\right)$ and also $\mathrm{H}_{2}(\mathrm{HL})$ states as effective twoand three-body problems. They also used correlated Gaussian results to check their calculations where possible. Moreover, they provide a qualitative sketch of the heteronuclear Efimov spectrum. We note, however, that this sketch is somewhat misleading concerning the window where dimeratom-atom Efimov states could form, as its size is exaggerated in light of our results. Comparing their results to ours, several differences can be noted.

From Fig. 2 in [35] we extracted their $c_{d} / c_{3}$ for $M / m=30$, which is roughly 0.03 , an order of magnitude larger than for our calculations (extrapolations via the effective three-body calculations to $M / m=30$ suggest something of order $10^{-3}$ ). In Ref. [35], separable potentials of Yamaguchi type between the $H L$ pairs were used [52]. So there are three obvious possible reasons for the discrepancy, a) $c_{d} / c_{3}$ is not universal and in fact depends on the $H L$ potential, b) the BO approximation is problematic in this region, as may be suggested by the fact that the scaling factor between consecutive Efimov states is 5.5 in their calculations, while the universal value is 3.96 [2], and c) the effective three-body calculations we used are not valid for higher mass ratios because of the larger $c_{d} / c_{3}$ value, and therefore the extrapolation to $M / m=30$ is not sound.

To rule out a), one would have to use a different potential and redo the calculations. Moreover, it is unclear whether the calculations of Wang et al. [35] are conducted closer to the unitary limit than ours, but since they state that $a_{H L} \gg r_{0}$ is not well satisfied in their calculations, we suppose $r_{0} / a_{H L}$ is of comparable size for both their calculations and ours (we have $r_{0} / a_{H L} \approx 1 / 4$ for the $(87 / 7)$ and $(133 / 6)$ systems, and $r_{0} / a_{H L} \approx 1 / 13$ for the $(7 / 6)$ system). In addition, because $c_{d} / c_{3}$ is very similar for the excited and ground state trimer in their calculation, and of roughly the same order of magnitude for different values of $r_{0} / a_{H L}$ in our calculations, a strong dependence on $r_{0} / a_{H L}$ is not likely.

Following the argument b), one might also add that our effective three-body calculation did not agree too well with our full four-body calculation, as shown in the previous section. (Note that the order of magnitude of $c_{d} / c_{3}$ is a fit parameter in our STM treatment, so it agrees by construction.) This might indicate that approximations that reduce the problem to an effective three-body system are generally insufficient to accurately describe the behavior near the dimer threshold.

Aspect c) should not play a huge role because the atom-dimer scattering length $a_{(H L) H}$ is estimated to be larger than $10^{7} r_{0}$ for $M / m=133 / 6$ and does not change much between $M / \mathrm{m}=$ $87 / 7 \approx 12$ and $M / m=133 / 6 \approx 22$, so extrapolating to $M / m=30$ should yield reasonable accuracy.

\section{B. The Scaling Factor}

The dimer-atom-atom Efimov effect is conjectured to arise when the four-body system of $H_{3} L$ can be treated as an effective three-body system that has an effective resonant interaction. This is expected to be the case in the vicinity where the $H_{2} L$ three-body state vanishes into the dimer threshold. There, Braaten and Hammer [2] argued, we can regard the $H L$ dimer as much more strongly bound than the trimer, allowing us to treat the trimer as an effective two-body system $(H L) H$ with an effective $(H L)-H$ scattering length $a_{(H L) H}$ which can be estimated by the inverse square root of the binding energy of the $\mathrm{H}_{2} L$ trimer. Then the $H_{3} L$ tetramer can be treated as an effective three-body system $\mathrm{H}_{2}(H L)$ which is also governed by the dimer-atom scattering length

$a_{(H L) H}$. For large $a_{(H L) H}$ this leads to the emergence of the Efimov effect. The scaling factor expected in this case is roughly 2000, because there are only two interacting pairs $(H L)-H$, 
and there are two "light" particles $H$ and one that is slightly heavier $(H L)$. The scenario with the smallest scaling factor is $m / M \rightarrow 0$, in which case it is 1986.1 [2]. This is the picture we have been using in our STM calculations shown in Fig. 6. Because of the huge scaling factor, we did not expect to be able to find these Efimov states with the accuracy we have reached.

However, in the Born-Oppenheimer approximation picture that Wang et al. [35] used, the light atom $L$ is seen as mediating the interaction between the heavy bosons $H$, leading to an effective three-body problem of three $H$ 's, with an effective scattering length $a_{H H}^{*}$. This effective scattering length diverges for the values of $a_{H L}$ where an Efimov trimer becomes unbound, so it shows the same behavior as $a_{(H L) H}$. But, since in this picture the $L$ is absorbed in the effective interaction, a symmetric system of three resonantly interacting $H$ 's emerges. The Efimov effect for this system, which is the well known three identical boson case, yields a much smaller scaling factor of $e^{\pi / s_{0}} \approx 22.7[2]$.

Using the Born-Oppenheimer approximation, Wang et al. [35] find somewhat tenuous evidence for an excited tetramer separated from the lower tetramer by a scaling factor of 14.3 on the positive $a_{H H}^{*}$ side and 19.3 on the negative side. This is in disagreement with the expectation from the EFT approach outlined above.

The main difference between the two approaches lies in the way the separation of scales is conducted. In the first approach, it is assumed that the $H L$ dimer is much more deeply bound than the $H_{2} L$ and $H_{3} L$ states, allowing a separation of energy scales and associated length scales. This assumption is found to be valid, because $\left|E_{D}\right| /\left|E_{3}^{0}-E_{D}\right|>300$ for the whole region shown in Figs. 4 and 5. On the other hand, in the BO approximation it is assumed that the time scale of the motion of the light atom is much shorter than that of the heavy atoms, allowing us to solve the dynamics of the light atom for fixed heavy atoms and then solving the heavy atoms system independently. This assumes that the light atom interacts with all the heavy atoms while they are fixed at some points, thus generating an effective interaction.

If however the light atom is very tightly bound to one heavy atom, as is the case here, its motion around the heavy atom might be on a shorter timescale than the motion of the heavy atom, but its motion towards the other heavy atoms is coupled to the motion of the heavy atom, which means it is on the same timescale and therefore not separable. So care would have to be taken to ensure that the light atom only induces an interaction of the heavy atom it is localised on with the other two heavy atoms, but not between the other two heavy atoms.

This means that in our understanding, both effective treatments are mutually exclusive and have to be applied to different systems. If we regard the two approximations as two ends of a spectrum, the BO approximation as used in [35] would apply to the case where the dimer and the trimer are both very weakly bound, and the EFT approximation would apply to the case where the dimer is infinitely strongly bound. Between these two points, we conjecture there is a transition between the three identical boson case with its relatively small scaling factor which then becomes larger and larger until it reaches the limit of two light bosons with one heavy boson. For the case we are studying here, this could mean that the scaling factor is slightly smaller than we would expect from our STM calculation. This however can only be explored using a full four-body calculation that is accurate enough to resolve these effective Efimov states.

\section{Resolution Estimate}

To estimate whether it would be possible to see the effective Efimov states in our calculations, we made some further assumptions. First, we assumed that the universal tetramer can be interpreted at the same time as the lowest state of the effective Efimov states. We do not expect this 
state to be fully universal, so the scaling factor between this state and the next may be smaller or larger, but we expect it to be of the same order of magnitude. This assumption was apparently also made by Wang et al. [35], although they did not mention it explicitly.

The second assumption is that the shape of the first excited effective Efimov state would resemble the shape of the universal tetramer near the threshold. Then we could scale the tetramer in Figs. 4 and 5 using different scaling factors to assess whether this state could be found with our accuracy. An example for a scaling factor of 20 is shown in Fig. 4.

Our four-body calculations presented here are by far not accurate enough to find effective Efimov states with a scaling factor of 2000. But our accuracy might just be sufficient to detect effective Efimov states if the scaling factor was indeed approximately 20 as suggested by Wang et al. [35]. We did not find any states, but since they would be just at the limit of our resolution, this is no conclusive evidence of their non-existence.

\section{SUMMARY AND OUTLOOK}

In this paper, we have presented a detailed study of the bound state spectrum of heteronuclear four-body systems of three heavy bosons $H$ and a light atom $L$ with resonant interspecies interaction in the region of positive scattering lengths. The interaction between the heavy bosons was assumed to be negligible. To obtain the energies of the $H_{2} L$ trimer and $H_{3} L$ tetramer systems, we employed the Gaussian expansion method (GEM) [39].

The general structure of the spectrum for different mass ratios was summarized in the Efimov plot of Fig. 3, a notable feature being that there is no excited Efimov tetramer at all for the mass ratio $M / m=7 / 6$. As the mass ratio increases, the excited state tetramer appears on the negative scattering length side of the Efimov plot. For $M / m=133 / 6$ it exists also in the unitary limit and disappears through the trimer-atom threshold on the positive scattering length side of the Efimov plot. Our results confirm and extend the findings of Blume and Yan [36], who conducted calculations down to $M / m=8 / 1$.

Next we focused on the dimer-atom(-atom) threshold region. We found that the value of the scattering length where the trimer vanishes into the threshold and the point where the tetramer does the same are extremely close to each other. We have investigated the dependence of the distance between these points, $c_{d}$, on the mass ratio $M / m$ and presented a detailed discussion of the sources of error in our calculation, such as a possible dependence of the results on the potential shape and missing higher partial wave contributions. We have estimated the size of errors from the latter to not be larger than $10^{-8} \sqrt{E_{s}}$ near the dimer-atom-atom threshold. The correlation between $c_{d}$ and $M / m$ does not appear to be linear, and the results from the four-body calculation could be reconciled only partly with effective three-body results in the framework of a STM-like equation derived from effective field theory [37]. We conclude that either an important aspect of the four-body system is missing in the effective three-body calculation close to the dimer-atomatom threshold, or the errors of our four-body results are underestimated. The resolution of this question requires further work, such as repeating the calculations for different potential shapes, to test the influence on $c_{d}$ and the general shape of the tetramer near the threshold. In order to rule out finite range effects a study of higher excited trimers and the tetramer resonances belonging to them would be useful.

In the last part, we discussed in depth the possibility of finding effective dimer-atom-atom Efimov states near the dimer-atom-atom threshold. We did not observe such states and estimated it is unlikely to find them without a major improvement of the accuracy in our calculation. Wang and collaborators [35] have previously found such states, albeit only in effective three-body calcula- 
tions within the Born-Oppenheimer approximation. We have compared our results to the results of [35] and highlighted some important differences between the two approaches. The key question in reconciling the results involves the scaling factor for the effective Efimov states. We expect a large scaling factor of order 2000 since only the $H L$ dimer and the $H$ atoms of the effective threebody system are interacting resonantly. We estimated that for this scaling factor an observation of the effective Efimov states in our calculation is very unlikely. In the Born-Oppenheimer picture, however, the light $L$ atom mediates an interaction between all three $H$ atoms such that there are effectively three resonant pair interactions and the scaling factor should be much smaller and close to 22.7. As we discussed in detail, it is not obvious that the Born-Oppenheimer picture is still applicable near the dimer-atom-atom threshold. Settling the question of the effective dimer-atomatom Efimov states in heteronuclear systems requires a significant increase in the accuracy of our four-body calculations and a coordinated study of the problem in both approaches.

\section{ACKNOWLEDGMENTS}

We acknowledge useful discussions with Doerte Blume on heteronuclear few-body systems and practical units, and with Artem Volosniev on the limitations of the Born-Oppenheimer approximation. We also thank Doerte Blume for providing data from her figures from [36]. This research was supported in part by the Deutsche Forschungsgemeinschaft through SFB 1245 and by the German Federal Ministry of Education and Research under contract 05P15RDFN1. C.H.S. thanks RIKEN for support under the IPA program and the Studienstiftung des deutschen Volkes for support under the RIKEN program. Some of the calculations were carried out at supercomputing facilities of the YITP (Kyoto University) and of Nagoya University.

\section{Appendix A: Effective field theory framework}

An effective field theory for heteronuclear three-body systems consisting of an atom of mass $m_{1}$ and two identical bosonic atoms of mass $m_{2}$ was developed by Helfrich et al. [37]. This effective field theory leads to an analog of the Skorniakov-Ter-Martirosian (STM) equation [53] for the unequal mass case. Similar equations also arise in the effective field theory description of twoneutron halo nuclei in nuclear physics [54]. Here we focus on the case of zero-range interactions between the unlike atoms characterized by a scattering length $a_{12}$ and no interaction between the like atoms $\left(a_{22} \equiv 0\right)$.

The trimer binding energies $B$ in this framework are given by the non-trivial solutions of the integral equation:

$$
\chi(p ; B)=\frac{m_{1}}{2 \pi \mu_{12}} \int_{0}^{\Lambda} d q \frac{q}{p} \ln \left(\frac{p^{2}+q^{2}+2 p q \mu_{12} / m_{1}+2 \mu_{12} B}{p^{2}+q^{2}-2 p q \mu_{12} / m_{1}+2 \mu_{12} B}\right) \frac{\chi(q ; B)}{-\frac{1}{a_{12}}+\sqrt{2 \mu_{12}\left(B+\frac{q^{2}}{2 \mu_{(12) 2}}\right)}},
$$

where $\mu_{12}=m_{1} m_{2} /\left(m_{1}+m_{2}\right)$ and $\mu_{(12) 2}=\left(m_{1}+m_{2}\right) m_{2} /\left(m_{1}+2 m_{2}\right)$ are the reduced masses of the 12 and (12)2 systems. The cutoff $\Lambda$ plays the role of the three-body parameter and can be used to fix the energy $B$ of any three-body state in the system. $]^{3}$ All other three-body energies can

\footnotetext{
${ }^{3}$ Note that in Eq. A1, the log-periodicity of the three-body force term in the effective field theory was used to move the dependence on the three-body parameter to the upper limit of the integral (see Ref. [55] for more details).
} 
then be predicted. Alternatively, the energies can be predicted in units of $\Lambda^{2}$. The wave function of the trimers can be obtained from the function $\chi(p ; B)$ but will not be required here.

[1] V. Efimov, Phys. Lett. 33B, 563 (1970).

[2] E. Braaten and H.-W. Hammer, Phys. Rept. 428, 259 (2006) [arXiv:cond-mat/0410417].

[3] L. Platter, Few Body Syst. 46, 139 (2009) [arXiv:0904.2227].

[4] P. Naidon and S. Endo, arXiv:1610.09805 [quant-ph].

[5] E. Nielsen and J.H. Macek, Phys. Rev. Lett. 83, 1566 (1999).

[6] B.D. Esry, C.H. Greene, and J.P. Burke, Phys. Rev. Lett. 83, 1751 (1999).

[7] P.F. Bedaque, E. Braaten, and H.-W. Hammer, Phys. Rev. Lett. 85, 908 (2000) [arXiv:condmat/0002365].

[8] E. Braaten and H.-W. Hammer, Phys. Rev. Lett. 87, 160407 (2001) [arXiv:cond-mat/0103331].

[9] E. Braaten and H.-W. Hammer, Phys. Rev. A 70, 042706 (2004) [arXiv:cond-mat/0303249].

[10] T. Kraemer, M. Mark, P. Waldburger, J.G. Danzl, C. Chin, B. Engeser, A.D. Lange, K. Pilch, A. Jaakkola, H.-C. Nägerl, and R. Grimm, Nature 440, 315 (2006) [arXiv:cond-mat/0512394].

[11] F. Ferlaino and R. Grimm, Physics 3, 9 (2010).

[12] R.D. Amado and J.V. Noble, Phys. Rev. D 5, 1992 (1972).

[13] V. Efimov, JETP Lett. 16, 34 (1972)

[14] V. Efimov, Nucl. Phys. A 210, 157 (1973).

[15] S.-K. Tung, K. Jimenez-Garcia, J. Johansen, C. V. Parker, C. Chin, Phys. Rev. Lett. 113, 240402 (2014) [arXiv:1402.5943 [cond-mat.quant-gas]].

[16] J. Johansen, B. J. DeSalvo, K. Patel, C. Chin, arXiv:1612.05169 [cond-mat.quant-gas].

[17] R. Pires, J. Ulmanis, S. Häfner, M. Repp, A. Arias, E.D. Kuhnle, M. Weidemüller, Phys. Rev. Lett. 112, 250404 (2014) [arXiv:1403.7246 [cond-mat.quant-gas]].

[18] J. Ulmanis, S. Häfner, R. Pires, E. D. Kuhnle, M. Weidemüller, and E. Tiemann, New J. Phys. 17, 055009 (2015) [arXiv:1501.04799 [cond-mat.quant-gas]].

[19] J. Ulmanis, S. Häfner, R. Pires, F. Werner, D. S. Petrov, E. D. Kuhnle, and M. Weidemüller, Phys. Rev. A 93, 022707 (2016) [arXiv:1509.05585 [cond-mat.quant-gas]].

[20] J. Ulmanis, S. Häfner, R. Pires, E. D. Kuhnle, Y. Wang, C. H. Greene and M. Weidemüller, Phys. Rev. Lett. 117, 153201 (2016) [arXiv:1608.01477 [cond-mat.quant-gas]].

[21] S. Häfner, J. Ulmanis, E. D. Kuhnle, Y. Wang, C. H. Greene, M. Weidemüller, arXiv:1701.08007 [cond-mat.quant-gas].

[22] B. Acharya, C. Ji and L. Platter, Phys. Rev. A 94, 032702 (2016) [arXiv:1606.04508 [cond-mat.quantgas]].

[23] L. Platter, H.-W. Hammer and U. G. Meißner, Phys. Rev. A 70, 052101 (2004) [cond-mat/0404313].

[24] A. Zenesini, B. Huang, M. Berninger, S. Besler, H.-C. Nägerl, F. Ferlaino, R. Grimm, C. H. Greene, and J. von Stecher, New Journal of Physics 15, 043040 (2013) [arXiv:1205.1921]

[25] H.-W. Hammer and L. Platter, Eur. Phys. J. A 32, 113 (2007) [nucl-th/0610105].

[26] J. von Stecher, J. D'Incao, and C. Greene, Nature Physics 5, 417 (2009) [arXiv:0810.3876].

[27] A. Deltuva, Phys. Rev. A 82, 040701 (2010) [arXiv:1009.1295 [physics.atm-clus]].

[28] A. Deltuva, Few-Body Systems 54, 569 (2013) [arXiv:1202.0167 [physics.atom-ph]].

[29] J. von Stecher, J. Phys. B: At. Mol. Opt. Phys. 43, 101002 (2010) [arXiv:0909.4056].

[30] M. Gattobigio, A. Kievsky and M. Viviani, Phys. Rev. A 86, 042513 (2012) [arXiv:1206.0854 [physics.atm-clus]]. 
[31] A. N. Nicholson, Phys. Rev. Lett. 109, 073003 (2012) [arXiv:1202.4402 [cond-mat.quant-gas]].

[32] A. Kievsky, N. K. Timofeyuk and M. Gattobigio, Phys. Rev. A 90, 032504 (2014) [arXiv:1405.2371 [cond-mat.quant-gas]].

[33] Y. Yan and D. Blume, Phys. Rev. A 92, 033626 (2015) [arXiv:1508.00081 [cond-mat.quant-gas]].

[34] F. Wang, X. Ye, M. Guo, D. Blume and D. Wang, ArXiv e-prints (Nov. 2016). [arXiv:1611.03198[cond-mat.quant-gas]]

[35] Y. Wang, W. B. Laing, J. von Stecher and B. D. Esry, Phys. Rev. Lett. 108, 073201 (2012) [arXiv:1109.1581[physics.atom-ph]].

[36] D. Blume and Y. Yan, Phys. Rev. Lett. 113, 213201 (2014) [arXiv:1410.2314[cond-mat.quant-gas]].

[37] K. Helfrich, H.-W. Hammer and D. S. Petrov, Phys. Rev. A 81, 042715 (2010) [arXiv:1001.4371 [cond-mat.quant-gas]].

[38] C. H. Schmickler, H.-W. Hammer and E. Hiyama, Few Body Syst. 58, 22 (2017).

[39] E. Hiyama, Y. Kino, and M. Kamimura, Prog. Part. Nucl. Phys. 51, 223 (2003).

[40] E. Hiyama, M. Kamimura, T. Motoba, T. Yamada, and Y. Yamamoto, Phys. Rev. C 53, 2075 (1996).

[41] E. Hiyama, M. Kamimura, T. Motoba, T. Yamada, and Y. Yamamoto, Prog. Theor. Phys. 97, 881 (1997).

[42] E. Hiyama, M. Kamimura, K. Miyazaki, and T. Motoba, Phys. Rev. C 59, 2351 (1999).

[43] E. Hiyama, M. Kamimura, T. Motoba, T. Yamada, and Y. Yamamoto, Phys. Rev. C 65, 011301(R) (2001).

[44] E. Hiyama, M. Kamimura, T. Motoba, T. Yamada, and Y. Yamamoto, Phys. Rev. C 66, 024007 (2002).

[45] E. Hiyama, M. Kamimura, Y. Yamamoto, and T. Motoba, Phys. Rev. Lett. 104, 212502 (2010).

[46] E. Hiyama and M. Kamimura, Phys. Rev. A 85, 022502 (2012).

[47] E. Hiyama and M. Kamimura, Phys. Rev. A 85, 062505 (2012).

[48] E. Hiyama and M. Kamimura, Phys. Rev. A 90, 052514 (2014).

[49] M. Gattobigio, A. Kievsky and M. Viviani, Phys. Rev. A 84, 052503 (2011) [arXiv:1106.3853 [physics.atm-clus]].

[50] D. Blume, private communication (2015).

[51] A. Deltuva, Phys. Rev. A 85, 042705 (2012) [arXiv:1203.6291].

[52] Y. Wang, private communication (2017).

[53] G. V. Skorniakov and K. A. Ter-Martirosian, Sov. Phys. JETP 4, 648 (1957).

[54] D. L. Canham and H.-W. Hammer, Eur. Phys. J. A 37, 367 (2008) [arXiv:0807.3258 [nucl-th]].

[55] H.-W. Hammer and T. Mehen, Nucl. Phys. A 690, 535 (2001) [nucl-th/0011024]. 